\title{
Validação de uma escala de mensuração de atitude em STEM em um contexto brasileiro na Universidade de Pernambuco utilizando análise fatorial confirmatória
}

\author{
Weverton F. de M. Melo ${ }^{1}$, Alexandre M. A. Maciel $^{1}$, Rodrigo L. Rodrigues ${ }^{2}$, Anu \\ A. Gokhale ${ }^{3}$ \\ ${ }^{1}$ Escola Politécnica (POLI) - Universidade de Pernambuco (UPE) - Pernambuco - PE, \\ Brasil \\ ${ }^{2}$ Programa de Pós-Graduação em Tecnologia e Gestão em Educação a Distância \\ (PPGTEG) - Universidade Federal Rural de Pernambuco - Pernambuco - PE, Brasil \\ ${ }^{3}$ Computer Systems Technology Program - Illinois State University, USA \\ \{wfmm, amam\}@ecomp.poli.br, rlrecin.ufpe.br, aagokha@ilstu.edu
}

\begin{abstract}
Resumo
O ensino de Ciência, Tecnologia, Engenharia e Matemática (STEM) dentro de todos os níveis educacionais está se tornando de grande importância em escala mundial, mas no Brasil os números indicam um déficit. Neste contexto, o objetivo deste artigo consiste em validar a aplicação de uma escala de atitude em relação a STEM dentro do contexto brasileiro. A amostra é composta por um grupo alunos ingressantes nos cursos de engenharia. A validação da escala foi feita com a utilização de análise fatorial confirmatória, na qual os resultados apontaram que $67 \%$ dos itens da escala eram compatíveis a realidade brasileira. Os outros itens foram alterados, gerando uma nova escala, adaptada ao cenário educacional STEM no Brasil.
\end{abstract}

Palavras-chave: Atitude sobre STEM, GMB-A-ST, Análise Fatorial Confirmatória, Validação de Escala, Ciência e Tecnologia.

\section{Validation of a STEM attitude measurement scale in a Brazilian context at the University of Pernambuco using a confirmatory factorial analysis}

\begin{abstract}
The teaching of Science, Technology, Engineering and Mathematics (STEM) at all levels of education is becoming of great importance worldwide, but in Brazil the numbers indicate a deficit. In this context, the purpose of this article is to validate the application of a scale of attitudes in relation to STEM in the Brazilian context. The sample is composed of a group of students entering the engineering courses. The validation of the scale was performed through a confirmatory factorial analysis, in which the results indicated that $67 \%$ of the scale items were compatible with the Brazilian reality. The other items were changed, generating a new scale, adapted to the educational scenario STEM in Brazil.
\end{abstract}

Keywords: Attitudes Toward STEM, GMB-A-ST, Confirmatory Factor Analysis, Validation of Scale, Science and Technology. 


\section{Introdução}

O termo STEM (Science, Technology, Engineering and Mathematics) refere-se ao ensino e aprendizagem nos campos da Ciência, Tecnologia, Engenharia e Matemática. Tipicamente, inclui atividades educacionais em todos os níveis - desde o pré-escolar até o pós-doutorado - cujo objetivo é proporcionar aos alunos estratégias cognitivas que lhes permitam pensar criticamente, tomar decisões e resolver problemas complexos (Gonzalez e Kuenzi, 2012). Aplica-se às mais diversas áreas que não envolvem apenas cursos relativos a tecnologia, buscando estimular seu público alvo a interagirem e perceberem a tecnologia que os rodeia.

A importância do seu ensino não está apenas em levar os estudantes a uma carreira significativa e gratificante, mas também permitir que as pessoas sejam cidadãs ativas em uma sociedade cada vez mais tecnológica. De acordo com o Departamento de Comércio dos Estados Unidos, os empregos STEM têm crescido três vezes mais rápidos do que do que os não-STEM. Na próxima década, eles vão crescer a uma taxa de $17 \%$ - em comparação com um crescimento previsto de 9,8\% em outras ocupações (White House, 2016; Gilibrand, e Kennedy III, 2014).

O Brasil não apresenta bons indicadores de formação de STEM, quando comparado a países desenvolvidos. O estado de Pernambuco, especificamente, apresenta indicadores ainda piores quando comparado com a realidade nacional. Um exemplo disto é que entre 2005 e 2015, apesar do indicador principal IDEB ter aumentado 44\%, as notas SAEB em matemática e português aumentaram somente $8 \%$ e $11 \%$, respectivamente (Basto-Filho, et al., 2016).

A situação fica ainda pior quando se constata uma alta demanda no setor de STEM, e a baixa formação de cientistas, principalmente de qualidade. Países como os Estados Unidos formam dez vezes mais; a França, três vezes mais; e o Brasil, por causa do baixo investimento e incentivo, forma menos comparado ao seu potencial e necessidade (Almeida, 2018). Para gerar inovação, é preciso formar mais cientista e engenheiros, e para isso, é preciso estimular os alunos a seguirem uma carreira em STEM (Senado, 2011).

Estes números projetam uma queda, que exige aos pesquisadores uma concentração em estudos que investiguem sobre a atitude dos estudantes em relação a ciência e tecnologia, para que o problema possa ser compreendido e reparado desde a sua base (Osborne,et al.,2003). Entretanto, mensurar uma definição sobre atitude em relação a área de STEM ainda é algo impreciso, e a quantidade de pesquisas que explanam sobre instrumentos que possam medir essa escala de atitude é bem escassa, principalmente na realidade brasileira.

Diante deste cenário, este artigo tem como objetivo realizar a validação e adaptação do instrumento GMB-A-ST (Gokhale, et al., 2013) na realidade brasileira, aplicando em alunos do primeiro ano de engenharia da Universidade de Pernambuco, utilizando análise fatorial.

Este artigo está estruturado da seguinte forma. Na seção 2 explicitamos as diferentes abordagens de mensuração de atitude que vem sendo desenvolvidas ao longo dos anos, bem como os diversos instrumentos de coleta que foram desenvolvidos. A seção 3 é descrito o método utilizado neste trabalho, características da amostra, instrumento de coleta, procedimentos e análise dos dados. A seção 4 é descrito a interpretação dos resultados e na seção 5 são relatadas as considerações finais. 


\section{Fundamentação Teórica}

Esta seção capítulo aborda os principais trabalhos relacionados, conceituando e contextualizando as variáveis necessárias para a compreensão do trabalho em questão.

\subsection{Avaliação da atitude dos estudantes em relação a STEM}

O conceito de atitude define de que é um sentimento positivo ou negativo sobre algum objeto social, que é organizado e assimilado através da experiência que influencia nas respostas afetivas e cognitivas de um indivíduo em relação a algum outro indivíduo, objeto ou evento (Nunnally, 1970 e Reid, 2006). O sentimento gera uma atitude que leva a intenções comportamentais, que influenciam o comportamento aplicado, que por sua vez, faz com o que o indivíduo remodele a sua atitude inicial (Fraser, 1982). Conforme as pessoas enriqueçam seu conhecimento e percepção sobre assuntos de ciência e tecnologia, desenvolvendo suas competências, e acostumando-se com suas descobertas, a atitude em relação a STEM tende a crescer e evoluir constantemente.

Há alguns anos, a comunidade de pesquisa educacional tem levado em consideração dentro de seus trabalhos, realizar uma investigação das atitudes dos alunos em relação a ciência e tecnologia. Um exemplo de instrumento foi desenvolvido por (Sandman, 1973) e posteriormente alterado por (Gogolin e Swartz, 1992) para avaliar as atitudes em relação a matemática. Com ênfase em analisar o ponto de vista dos alunos sobre a natureza da ciência foi desenvolvido o The View on Science-Technology-Society (Aikenhead, et al., 1989). Este último citado, foi adaptado para descobrir os perfis de alunos de graduação de química com opiniões positivas ou negativas sobre química (Bennett, 2001). Para analisar a atitude em relação a disciplina de Física Geral (Da Silveira, 1979).

Atualmente, a importância destes estudos tem sido ainda mais relevante pela queda no interesse em campos como ciência, tecnologia, engenharia e matemática (denominados "STEM"), que são a base da futura evolução tecnologia (National Science Foundation, 2002).

Neste momento, os Estados Unidos é o país líder mundial no desenvolvimento científico e tecnológico, mas outros países, como Canadá, Inglaterra, Alemanha, estão prontos para desafiar sua potência. Este desafio é acentuado por um déficit projetado de cientistas e engenheiros na força de trabalho. Nas próximas décadas, a demanda por essas profissões deverá aumentar em $47 \%$, enquanto a força de trabalho aumentará apenas 15\% (Bureau of Labor Statistics, 2002). O Brasil emprega mais de 1,3 milhão de pessoas no setor, e nos próximos quatro anos tem uma estimativa de que irá precisar de 750 mil profissionais. Mas a baixa quantidade de profissionais formando-se, além do mais, de qualidade insuficiente, não supriram as necessidades mercadológicas (Knebel, 2016).

Consequentemente, este frequente declínio na quantidade de alunos que escolhem estudar ciência e tecnologia, exige um foco de pesquisa nas atitudes dos alunos em relação as mesmas, para que a natureza do problema seja entendida e remediada. No entanto, o conceito de uma atitude em relação à ciência e à tecnologia é algo pouco explorado e, portanto, pode ser difícil de mensurar.

Acompanhando está situação, (Gokhale, et al., 2013) após mais de uma década de estudos, desenvolveram um questionário, composto por 30 questões que busca medir a atitude do estudante em relação a ciência e tecnologia. As questões abordam alguns assuntos principais, como, o interesse na obtenção de conhecimento científico e 
tecnológico; a ciência e tecnologia são benéficas para a humanidade; ciência e tecnologia é uma área para mulheres; homens e mulheres têm igual oportunidade em ciência e tecnologia; e a preocupação com os pontos perigosos do desenvolvimento científico e tecnológico. $\mathrm{O}$ resultado deste questionário demonstra não só o nível de atitude, mas também, em quais pontos está atitude é mais positiva ou negativa, facilitando no entendimento da vivência e ponto de vista dos estudantes em relação a STEM.

\section{Materiais e Métodos}

Para atingir o objetivo de validar um instrumento de coleta de dados sobre a escala de atitude em relação à ciência e tecnologia foi utilizado a técnica mais adequada para verificar a validade de um instrumento, que é a Análise Fatorial Confirmatória (AFC) (Gable, 1986).

\subsection{Instrumento de Coleta}

O instrumento de coleta utilizado nesta pesquisa foi adaptado do questionário GokhaleMachina-Brauchle de Atitudes em Relação à Ciência e Tecnologia (GMB-A-ST) desenvolvido por Gokhale, et al., 2013. Este instrumento é composto por 30 itens com um formato de resposta do tipo escala de Likert (Guilford, 1975) com 5 para cada item da pesquisa - 'concordo total (5) / concordo parcial (4) / neutro (3) / discordo parcial (2) / discordo total (1)' - para indicar seus próprios sentimentos (Quadro 1, Quadro 2) com o intuito de avaliar a atitude em relação as áreas de STEM.

O 'concordo' foi classificada como cinco pontos para itens formuladas positivamente e um ponto para itens formuladas de forma negativa, 'concordo' foi marcado como quatro pontos para itens formuladas positivamente e dois pontos para itens formuladas de forma negativa, 'neutro' foi marcado como três pontos , 'discordo' foi marcado como dois pontos para itens formuladas positivamente e quatro pontos para itens formuladas de forma negativa, e 'discordo' foi marcado como um ponto para itens formuladas positivamente e cinco pontos para itens formuladas negativamente. Consequentemente, uma pontuação elevada representa uma atitude positiva; uma pontuação baixa representa uma atitude negativa.

Quadro 1. Variáveis do questionário de atitude em STEM.

\begin{tabular}{|c|l|}
\hline $\mathbf{N}$ & Item do questionário \\
\hline 1 & $\begin{array}{l}\text { A ciência e a tecnologia estão tornando nossas vidas mais saudáveis, mais fáceis e mais } \\
\text { confortáveis }\end{array}$ \\
\hline 2 & Os homens tendem a ter mais talento natural para a ciência e tecnologia do que as mulheres \\
\hline 3 & Em geral, os computadores e a automação de fábrica criam mais empregos do que eliminam \\
\hline 4 & É importante para mim, no meu dia a dia, conhecer a ciência \\
\hline 5 & As mesmas oportunidades de sucesso em áreas técnicas estão disponíveis para homens e mulheres \\
\hline 6 & A tecnologia faz o nosso modo de vida mudar muito rápido \\
\hline 7 & Por causa da ciência e tecnologia, o trabalho se torna mais atraente \\
\hline 8 & $\begin{array}{l}\text { Ambientes familiares são mais disponíveis nas profissões de ciência e tecnologia do que em } \\
\text { outras }\end{array}$ \\
\hline 9 & As descobertas tecnológicas estão destruindo a terra \\
\hline 10 & $\begin{array}{l}\text { Cursos de ciência e tecnologia fazem contribuições significativas para a educação e formação de } \\
\text { uma pessoa }\end{array}$ \\
\hline 11 & $\begin{array}{l}\text { As mesmas oportunidades para desenvolver habilidades técnicas estão disponíveis para homens } \\
\text { e mulheres }\end{array}$ \\
\hline 12 & Por causa da ciência e tecnologia, haverá mais oportunidades para a próxima geração \\
\hline 13 & Os benefícios da pesquisa científica e tecnológica tem superado os prejuízos que ela trás \\
\hline
\end{tabular}




\begin{tabular}{|l|l|}
\hline 14 & É bom para as mulheres ter uma carreira em ciência e tecnologia \\
\hline 15 & As pessoas fariam o certo se escolhessem viver uma vida mais simples, sem usar tanta tecnologia \\
\hline 16 & O desenvolvimento tecnológico cria um modo de vida artificial e desumano \\
\hline 17 & $\begin{array}{l}\text { O ambiente de trabalho enfrentado pelas mulheres em ciência e tecnologia é o mesmo que o } \\
\text { enfrentado pelos homens }\end{array}$ \\
\hline 18 & A maioria dos cientistas quer trabalhar em coisas que irão tornar melhor a vida das pessoas \\
\hline 19 & As mulheres devem ser incentivadas a seguirem carreiras em ciência e tecnologia \\
\hline 20 & São os homens e não as mulheres devem desempenhar papéis de liderança em ciência e tecnologia \\
\hline 21 & $\begin{array}{l}\text { Novas invenções serão sempre descobertas para neutralizar qualquer consequência prejudicial do } \\
\text { desenvolvimento tecnológico }\end{array}$ \\
\hline 22 & $\begin{array}{l}\text { Mesmo que não traga benefícios imediatos, a pesquisa científica e tecnológica devem ser apoiadas } \\
\text { pelo governo federal }\end{array}$ \\
\hline 23 & Gosto de aprender sobre novas descobertas científicas e seus efeitos sobre a tecnologia \\
\hline 24 & Estou bem informado sobre novas invenções e tecnologias \\
\hline 25 & Estou interessado na aplicação da ciência e tecnologia na exploração espacial \\
\hline 26 & Gosto de ler sobre ciência, matemática, engenharia e tecnologia \\
\hline 27 & Gosto de ver programas/conteúdos de ciência e natureza na televisão \\
\hline 28 & Procuro informações sobre temas de ciência, tecnologia, engenharia ou matemática na internet \\
\hline 29 & $\begin{array}{l}\text { Devemos construir um centro de inovação tecnológica grande o suficiente para abrigar } \\
\text { experimentos científicos e de fabricação }\end{array}$ \\
\hline 30 & As mulheres teriam mais êxito em ciência e tecnologia se existisse um forte sistema de apoio \\
\hline
\end{tabular}

Quadro 2. Variáveis divididas em seus fatores.

\begin{tabular}{|l|c|}
\hline Fatores & Variáveis \\
\hline Interesse na obtenção de conhecimento em Ciência e Tecnologia & $\begin{array}{c}\text { 4, 10, 23, 24, 25, 26, } \\
27,28\end{array}$ \\
\hline Ciência e Tecnologia são benéficos para a humanidade & $1,3,7,12,13,21$ \\
\hline Adequação da Ciência e Tecnologia para as Mulheres & $2,14,19,20,30$ \\
\hline $\begin{array}{l}\text { Os homens e as mulheres têm igualdade de oportunidades em Ciência e } \\
\text { Tecnologia }\end{array}$ & $5,11,17$ \\
\hline A preocupação de que Ciência e Tecnologia é perigoso para a humanidade & $6,9,15,16$ \\
\hline *Sem fator & $29,22,18,8$ \\
\hline
\end{tabular}

*Itens inclusos de forma aleatória, apenas para teste pelos desenvolvedores da GMB-A-ST.

Apresentamos com este artigo a adaptação e validação deste instrumento em contexto da educação brasileira. $\mathrm{O}$ objetivo foi analisar a adequação do instrumento resultante para coletar o ponto de vista que indicará a atitude do aluno em relação a STEM. A próxima seção descreve as características da amostra coletada.

\subsection{Público Alvo e Amostra}

Para a execução da Análise Fatorial Confirmatória (AFC), com o intuito de validação do instrumento de coleta GMB-A-ST, foi utilizada uma amostra coletada através da aplicação do instrumento no campus Escola Politécnica de Pernambuco (POLI) da Universidade de Pernambuco (UPE) que oferece cursos de graduação, especialização e pós-graduação.

A aplicação foi realizada na aula magna, com os ingressantes de todos os cursos das engenharias na graduação, sendo os de Engenharia Civil, da Computação, Elétrica Eletrônica, Elétrica Eletrotécnica, Elétrica Telecomunicações, Mecânica, e de Controle de Automação, totalizando uma amostra de 165 alunos.

\subsection{Análise e interpretação dos dados}

Para as análises deste artigo foi utilizado o modelo SEM (structural equation model) desenvolvido por (Rodrigues, et al., 2016) utilizando o software estatístico R para validar 
uma escala de autorregulação da aprendizagem em ambiente de aprendizagem online com dados de estudantes brasileiros.

Neste modelo SEM, foram calculados os valores do Alpha de Cronbach para cada um dos itens da escala de atitude em STEM. Este índice é utilizado para verificar a fidelidade e medir a consistência da escala, isto é, para avaliar a magnitude em que os itens de um instrumento estão correlacionados, sendo uma média das correlações entre os itens que fazem parte de um instrumento (Cronbach, 1971).

A Análise Fatorial Confirmatória (AFC) foi demonstrada em forma de diagrama ao final do modelo SEM, para a identificação dos itens que compartilham variação suficiente para justificar a sua permanência ou remoção da escala.

\section{Resultados e Discussão}

Primeiramente o modelo SEM, foi especificado com os itens e fatores baseado na escala de mensuração de atitude em STEM, desenvolvido por (Gokhale, et al., 2013). A Figura 1 demonstra como foi estabelecido a estruturação para cada um dos cinco fatores, e seus respectivos itens, em script no software $\mathrm{R}$.

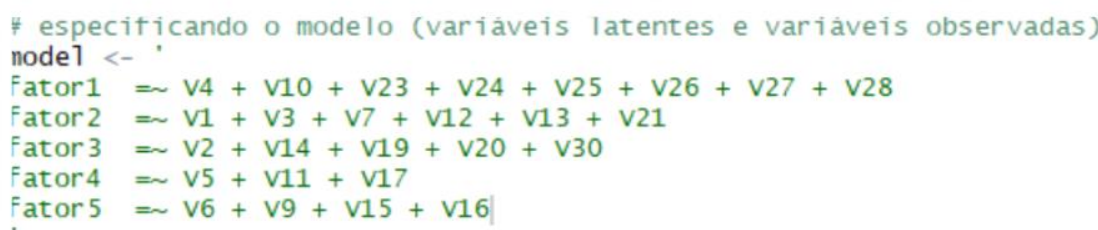

Figura 1. Especificando o Modelo.

O fator1 representa os itens que compõem 'Interesse na obtenção de conhecimento em Ciência e Tecnologia', o fator2 representa os itens que compõem a 'Ciência e Tecnologia são benéficos para a humanidade', o fator3 representa as 'Adequação da Ciência e Tecnologia para as Mulheres', o fator4 representa o 'Os homens e as mulheres têm igualdade de oportunidades em Ciência e Tecnologia', e por fim, o fator5 representa as 'A preocupação de que Ciência e Tecnologia é perigoso para a humanidade'. Os itens sem fatores, que foram inseridos por (Gokhale, et al., 2013) de forma aleatória, para teste, não foram inseridos.

Em seguida, após a execução do modelo SEM, a Análise Fatorial Confirmatória (AFC) é representada por um diagrama de caminho (Path Diagram), que demonstra de forma efetiva as relações entra as variáveis observadas e as variáveis latentes, da mesma maneira que os valores do Alpha de Cronbach de cada item.

Em termos de avaliação destes parâmetros, usamos as recomendações de (Comrey, 1973) que estabelecem valores iguais ou superiores a 0,71 - excelentes, 0,63 - muito bons, 0.55 - bons, 0,4 - justos, e 0,32 - pobres. Mesmo que algumas pesquisas aceitem cargas próximas do 0,3 , este estudo exigiu cargas fatoriais de pelo menos 0,4 para que um item seja considera relevante, e permaneça no instrumento.

A Figura 2 mostra o diagrama de caminho plotado, com os fatores (variáveis latentes) e os itens do questionário (variáveis observadas). 


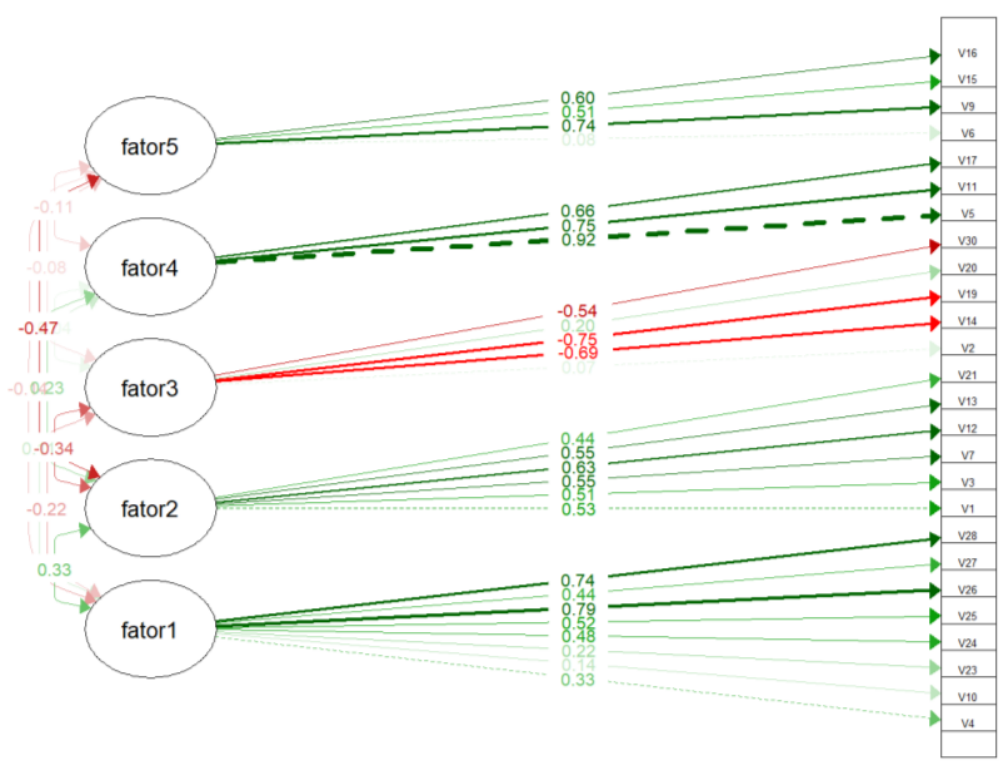

Figura 2. Diagrama de Caminho plotado.

A análise da Figura 2, com a plotagem do diagrama de caminho, finalizando a análise fatorial, revelou que maioria das variáveis observadoras (itens do questionário) estão acima do coeficiente estabelecido de 0,4 , estando validadas com a aplicação. Os itens v30, v19, v14, pertencentes ao fator 'Adequação da Ciência e Tecnologia para as Mulheres', que apesar de terem sido negativos, estão acima do coeficiente mínimo, o sinal não é considera na análise.

Entretanto, as variáveis observadoras v2, v4, v6, v10, v20 e v23, ficaram abaixo do coeficiente mínimo estabelecido de 0,4 , sendo consideradas de consistência interna baixa, e, portanto, foram removidas do questionário, juntamente com as variáveis que não tinham fator $(29,22,18,8)$, totalizando dez questões.

A partir dessas alterações, foi desenvolvido um novo fator com dez novas variáveis chamado de 'Experiências sociais e educacionais em relação a STEM' (Quadro 3, Quadro 4), com o intuito de verificar a vivência do aluno com ciência e tecnologia.

Quadro 3. O questionário alterado com as novas variáveis em relação a atitude em STEM.

\begin{tabular}{|c|l|}
\hline $\mathbf{N}$ & Item do questionário \\
\hline 2 & Estudei em um curso específico de preparação para cálculo antes da graduação \\
\hline 4 & $\begin{array}{l}\text { No ambiente escolar (ensino médio) pratiquei/desenvolvi experimentos em laboratórios de } \\
\text { ciência }\end{array}$ \\
\hline 6 & Meu conhecimento na língua estrangeira inglês é suficiente para comunicação \\
\hline 8 & $\begin{array}{l}\text { A escolha de um curso em ciência e tecnologia está relacionado com a minha expectativa de } \\
\text { ascensão social }\end{array}$ \\
\hline 10 & Durante o ensino médio participei de algum curso técnico \\
\hline 18 & Tive contato com aulas de robótica durante todo o ensino médio \\
\hline 20 & $\begin{array}{l}\text { Meu desempenho em disciplinas escolares exatas (matemática, física e química) foi entre bom e } \\
\text { excelente }\end{array}$ \\
\hline 22 & $\begin{array}{l}\text { Acredito que dificuldades financeiras podem influenciar negativamente no desenvolvimento de } \\
\text { cursos na área de exatas }\end{array}$ \\
\hline 23 & \begin{tabular}{l} 
Participei de curso de programação durante o ensino médio \\
\hline 29
\end{tabular} $\begin{array}{l}\text { O ciclo social (amigos e familiares) influenciou na minha escolha do curso que estudarei na } \\
\text { graduação da universidade }\end{array}$ \\
\hline
\end{tabular}


Quadro 4. Variáveis divididas em seus fatores alterados.

\begin{tabular}{|l|c|}
\hline Fatores & Variáveis \\
\hline Interesse na obtenção de conhecimento em Ciência e Tecnologia & $24,25,26,27,28$ \\
\hline Ciência e Tecnologia são benéficos para a humanidade & $1,3,7,12,13,21$ \\
\hline Igualdade entre mulheres e homens dentro da Ciência e Tecnologia & $5,11,14,17,19,30$ \\
\hline A preocupação de que Ciência e Tecnologia é perigoso para a humanidade & $9,15,16$ \\
\hline Experiências sociais e educacionais em relação a STEM & $2,4,6,8,10,18,20$, \\
$22,23,29$
\end{tabular}

Para que pudesse ser mantido a quantidade de cinco fatores na escala de mensuração de atitude em relação a STEM, após a inclusão do novo fator, os fatores 'Adequação da Ciência e Tecnologia para as Mulheres', e o 'Os homens e as mulheres têm igualdade de oportunidades em Ciência e Tecnologia' foram mesclados por serem semelhantes em seu tema, e tornaram-se 'Igualdade entre mulheres e homens dentro da Ciência e Tecnologia'.

O objetivo do presente estudo foi alcançado quando foi realizada uma Análise Fatorial Confirmatória (AFC) do da escala de mensuração de a percepção em ciência e tecnologia em um contexto brasileiro. $\mathrm{O}$ resultado permitiu a validação e o desenvolvimento do instrumento para a aplicabilidade da escala em pesquisas que queiram mensurar, através de questionário, a atitude de alunos em relação a STEM.

\section{Conclusão}

O presente estudo visou investigar a aplicabilidade da escala para mensurar a atitude em relação a STEM no contexto da realidade educacional do Brasil.

Os resultados deste estudo confirmaram a validação da escala GMB-A-ST para o contexto educacional no Brasil, em cima da amostra selecionada. Apesar de algumas variáveis terem consistência interna baixa, verificamos que o instrumento, desenvolvido e validado com universidades localizadas no Estados Unidos, em sua maioria, obteve indicadores de ajustes próximos em uma amostra com alunos no Brasil.

Com base nos resultados podemos afirmar que a escala é adequada para mensuração da atitude em relação a STEM em contextos educacionais brasileiros. Podendo ser adaptado e desenvolvido através de mais aplicações.

Como trabalhos futuros pretendemos validar e evoluir as novas variáveis incluídas, e a partir delas, a construção de perfis de estudantes, realizando a ligação entre as variáveis do questionário e a medida de desempenho dos alunos nos cursos de áreas de STEM, como forma de inferir resultados futuros dos estudantes, baseado nas suas atitudes sobre ciência e tecnologia durante o curso. 


\section{Referências Bibliográficas}

ALMEIDA, C. Sem cientistas de ponta, Brasil fica fora de cadeias globais. O Globo, São Paulo. 08 mar. 2018. Disponível em: < https://oglobo.globo.com/economia/semcientistas-de-ponta-brasil-fica-fora-de-cadeias-globais-22454291> Acesso em 17 mai. 2018.

AIKENHEAD, G., Ryan, A. G., \& Fleming, R. W. Views on science-technologysociety. Saskatoon, Saskatchewan, Department of Curriculum Studies, 1989.

BASTO-FILHO, C., GOKHALE, A., \& CAMPELLO, B. Relatório: Análise Preliminar e Sugestões para Criação de um Plano para Ampliação e Aperfeiçoamento do Ensino de STEM+C em Pernambuco. Relatório Técnico da Secretaria de Ciência, Tecnologia e Inovação de Pernambuco. 2016.

BENNETT, J. The development and use of an instrument to assess students' attitude to the study of chemistry. International Journal of Science Education, 2001. 23, p. 833-845.

BUREAU OF LABOR STATISTICS. Occupational Outlook Handbook. 2002. Disponível em: <http://www.bls.gov/oco/home.htm> Acesso em 17 mai. 2018.

CRONBACH, L.J. Coefficient alpha and internal structure of tests. Psychometrika. 1971, 15, 297-334.

DA SILVEIRA, F. L. Construção e validação de uma escala de atitude em relação a disciplinas de Física Geral. Revista Brasileira de Física, 1979. v. 9, n. 3.

GABLE, R. K. Instrument development in the affective domain. Boston, Massachuseets: Kluwer-Nijhoff Publishing, 1986.

GILIBRAND, K., KENNEDY III, J. STEM jobs key to better economy: Column. Usa Today, Janeiro, 2014. Disponível em: $<$ http://www.usatoday.com/story/opinion/2014/01/10/engineering-mathematics-stemgillibrand-kennedy-column/4361837/.> Acesso em 17 mai. 2018.

GOGOLIN, L., \& SWARTZ, F. A quantitative and qualitative inquiry into the attitudes toward science of nonscience college majors. Journal of Research in Science Teaching, 1992. 29, p. 487-504.

GONZALEZ, H. B., KUENZI, J. J. Science, Technology, Engineering, and Mathematics (STEM) Education: A Primer. Congressional Research Service, Agosto, 2012. Disponível em: <https://fas.org/sgp/crs/misc/R42642.pdf> Acesso em 17 mai. 2018.

GOKHALE, A., BRAUCHLE, P. E., MACHINA, K. F. Scale to measure attitudes toward information technology. International Journal of Information and Communication Technology Education (IJICTE), v. 9, n. 3, p. 13-26, 2013.

GUILFORD, J. P. Psychometric Methods. Tata MacGraw-Hill Co.Ltd, New York, 1979. $2 \mathrm{a}$.

KNEBEL, P. Novas tecnologias desafiam mercado de trabalho da TI. Jornal do Comércio, Porto Alegre. 16 nov. 2016. Disponível em: <http://jcrs.uol.com.br/_conteudo/2016/11/economia/531409-novas-tecnologiasdesafiam-mercado-de-trabalho-da-ti.html> Acesso em 20 mai. 2018. 
NATIONAL SCIENCE FOUNDATION. Science and engineering indicators - 2002, Division of Science Resources Statistics (NSB 02-01). 2002.

NUNNALLY, J.C. Introduccion a la Medicion Psicologica. Editorial Paidos. Buenos Aires, 1970. p. 469.

OSBORNE, J., SIMON, S., \& COLLINS, S. Attitudes towards science: a review of the literature and its implications. International Journal of Science Education. 1049-1079, 2003.

REID, N. Reflexões sobre a medição atitude. Pesquisa em Ciência e Educação Tecnológica, 2006. 24 (1), p. 3-27.

RODRIGUES, R. L. et al. Validação de um instrumento de mensuração de autorregulação da aprendizagem em contexto brasileiro usando análise fatorial confirmatória. RENOTE. 2016, v. 14, n. 1.

SANDMAN, R. S. The development, validation, and application of a multidimensional mathematics attitude instrument. Unpublished doctoral thesis (Minnesota: University of Minnesota), 1973.

SENADO. Formação em engenharia no Brasil: engenheiros para ciência e inovação tecnológica. Em Discussão!. Seminário CCT do Senado. 2011. Disponível em: $<$ https://www.senado.gov.br/noticias/Jornal/emdiscussao/inovacao/ensino-formacaoem-engenharia-no-brasil-faltam-engenheiros-ciencia-e-inovacao-tecnologica.aspx $>$. Acesso em 17 mai. 2018.

WHITE HOUSE. Progress Report on Coordinating Federal Science, Technology, Engineering, and Mathematics (STEM) Education. Março, 2016. Disponível em: $<$ https://www.whitehouse.gov/sites/default/files/microsites/ostp/stem_budget_suppleme nt_fy_17_final_0.pdf $>$ Acesso em 17 mai. 2018. 